\title{
Intracellular, In Vivo, Dynamics of Thalamocortical Synapses in Visual Cortex
}

\author{
Madineh Sedigh-Sarvestani, Leif Vigeland, Ivan Fernandez-Lamo, ${ }^{\circledR}$ M. Morgan Taylor, Larry A. Palmer, \\ and Diego Contreras \\ Department of Neuroscience, Perelman School of Medicine, University of Pennsylvania, Philadelphia, Pennsylvania 19403
}

Seminal studies of the thalamocortical circuit in the visual system of the cat have been central to our understanding of sensory encoding. However, thalamocortical synaptic properties remain poorly understood. We used paired recordings, in the lateral geniculate nucleus (LGN) and primary visual cortex (V1), to provide the first in vivo characterization of sensory-driven thalamocortical potentials in V1. The amplitudes of EPSPs we characterized were smaller than those previously reported in vitro. Consistent with prior findings, connected LGN-V1 pairs were only found when their receptive fields (RFs) overlapped, and the probability of connection increased steeply with degree of RF overlap and response similarity. However, surprisingly, we found no relationship between EPSP amplitudes and the similarity of RFs or responses, suggesting different connectivity models for intracortical and thalamocortical circuits. Putative excitatory regular-spiking (RS) and inhibitory fast-spiking (FS) V1 cells had similar EPSP characteristics, showing that in the visual system, feedforward excitation and inhibition are driven with equal strength by the thalamus. Similar to observations in the somatosensory cortex, FS V1 cells received less specific input from LGN. Finally, orientation tuning in V1 was not inherited from single presynaptic LGN cells, suggesting that it must emerge exclusively from the combined input of all presynaptic LGN cells. Our results help to decipher early visual encoding circuits and have immediate utility in providing physiological constraints to computational models of the visual system.

Key words: EPSP; thalamocortical

\section{Significance Statement}

To understand how the brain encodes the visual environment, we must understand the transfer of visual signals between various regions of the brain. Therefore, understanding synaptic dynamics is critical to our understanding of sensory encoding. This study provides the first characterization of visually evoked synaptic potentials between the visual thalamus and visual cortex in an intact animal. To record these potentials, we simultaneously recorded the extracellular potential of presynaptic thalamic cells and the intracellular potential of postsynaptic cortical cells in input layers of primary visual cortex. Our characterization of synaptic potentials in vivo disagreed with prior findings in vitro. This study will increase our understanding of thalamocortical circuits and will improve computational models of visual encoding.

\section{Introduction}

The visual thalamus, known as the lateral geniculate nucleus (LGN), is an obligatory relay of visual information to neocortex (Jones, 1985), and convergence of thalamic inputs gives rise to new functional properties in primary visual cortex (V1) layer 4

Received 0ct. 31, 2016; revised Feb. 24, 2017; accepted April 1, 2017.

Author contributions: M.S.-S., L.A.P., and D.C. designed research; M.S.-S., L.V., I.F.-L., M.M.T., L.A.P., and D.C. performed research; M.S.-S. analyzed data; M.S.-S., L.A.P., and D.C. wrote the paper.

This work was supported by National Institutes of Health Grant T32 NS091006 to M.S.-S., Grant F32 EY026463 to M.S.-S., and Grant R01 EY020765 to L.A.P. and D.C. We thank Gabe Murphy for comments on an earlier version of the manuscript.

The authors declare no competing financial interests.

Correspondence should be addressed to Dr. Madineh Sedigh-Sarvestani, Department of Neuroscience, Perelman School of Medicine, University of Pennsylvania, Philadelphia, PA 19403. E-mail: msarvestani@gmail.com. DOI:10.1523/JNEUROSCI.3370-16.2017

Copyright $\odot 2017$ the authors $\quad 0270-6474 / 17 / 375250-13 \$ 15.00 / 0$
(L4) neurons that are absent in the thalamus. To understand sensory representation in neocortex, we must first understand how response properties of cortical neurons are shaped by their thalamic inputs. Neurons in both the LGN and V1 are characterized by their receptive field (RF), the location and pattern of light increments or decrements that best drive the neuron. Hubel and Wiesel (1962) proposed that the RFs of simple cells in L4 of V1 results from the precise alignment of their afferent LGN inputs, a hypothesis supported by extracellular recording studies of geniculocortical (LGN-V1) cell pairs (Tanaka, 1983; Reid and Alonso, 1995; Alonso et al., 2001).

These studies provided the rules of connectivity between LGN and V1, which were based on the similarity of their respective RFs. Connectivity was shown to be dependent on various metrics but most notably the similarity in the preferred stimulus contrast of the RFs and the degree of RF overlap (Alonso et al., 2001). 
However, because the recordings were made extracellularly, these studies could not discard the possibility of weak subthreshold connections between cells with little RF overlap. The presence of these connections would completely change the rules of connectivity in the thalamocortical system. One goal of this study was to determine whether these connections exist. A second goal was to characterize the synaptic potentials between LGN and V1.

We obtained simultaneous recordings of extracellular spikes from LGN neurons and intracellular potentials from thalamorecipient neurons in V1 L4 and L6 of cats in vivo. In agreement with previous extracellular studies, we found that LGN-V1 cell pairs with similar RFs have higher connection likelihood. We did not find evidence for weak subthreshold connections between cells with poorly overlapped RFs. Consistent with this, but surprising given existing literature, we did not find a correlation between RF or response similarity and EPSP amplitude. The mean monosynaptic EPSP amplitude was several-fold smaller than the amplitude of putative geniculocortical EPSPs recorded in vitro (Stratford et al., 1996). Furthermore, EPSP amplitudes were similar for connections onto regular-spiking (RS) putatively excitatory and fast-spiking (FS), putatively inhibitory cells, in contrast to somatosensory cortex in which EPSP amplitudes are larger in FS cells. However, we did find some differences in RF similarity for LGN cells connected to RS V1 and those connected to FS V1 cells. Finally, we did not find a relationship between orientation bias of LGN cells and the optimal orientation of their target V1 cell, adding to our understanding of the differences between the emergence of orientation tuning in mouse and cat. Our study provides the first intracellular characterization of the thalamocortical EPSPs in cat V1, six decades after Hubel and Wiesel's exploration of this canonical circuit (Hubel and Wiesel, 1962).

\section{Materials and Methods}

Experiments were conducted according to the guidelines of the National Institutes of Health and with the approval of the Institutional Animal Care and Use Committee of the University of Pennsylvania.

Surgery and anesthesia. Surgical protocols are similar to those previously published by our group (Contreras and Palmer, 2003; Cardin et al., $2010)$. Adult male cats $(2.5-3.5 \mathrm{~kg})$ were anesthetized with an intraperitoneal injection of Nembutal $(25 \mathrm{mg} / \mathrm{kg})$. After completion of surgical procedures but before onset of visual stimuli, the animal was paralyzed with gallamine triethiodide (Flaxedil) and artificially ventilated (end tidal $\mathrm{CO}_{2}$ held at $4.0 \%$ ). Anesthesia was maintained by continuous infusion of sodium thiopental $(3-10 \mathrm{mg} / \mathrm{kg} / \mathrm{h})$ for some experiments $(n=$ $40)$, and propofol $(3-4 \mathrm{mg} / \mathrm{kg} / \mathrm{h})$ for others $(n=4)$. A pattern of EEG without predominant slow oscillations was maintained throughout the experiment. The EEG showed occasional spindles intermingled with desynchronized activity (Steriade et al., 1993). Vitals were monitored and rectal temperature was maintained at $37^{\circ} \mathrm{C}$.

Paired recordings. Two craniotomies and durotomies were made in the left hemisphere, at Horsley-Clarke coordinates [A6, L8] for the dorsal LGN (lamina A), and [P4, L2] for V1. The stability of the recordings was improved by bilateral pneumothorax, draining the cisterna magna, suspending the hips, and filling the cranial defect with 3.5\% agar. Glass pipettes (tip resistance between 50 and $90 \mathrm{M} \Omega$ ), filled with $3 \mathrm{~m}$ potassium acetate, were used for intracellular recordings in V1. Pipettes were lowered perpendicular to the surface of the apex of the lateral gyrus using a Kopf micropositioner. L4 simple cells were consistently found between 500 and $950 \mu \mathrm{m}$, as measured from the micropositionter. Recordings were made using a bridge amplifier (Neurodata) and digitized at $33 \mathrm{kHz}$ using a Neuralynx acquisition system. All cells used in this study had a stable $V_{\mathrm{m}}<-60 \mathrm{mV}$, had overshooting action potentials, and were observed for at least $10 \mathrm{~min}$.

Following surgery, an array of 5-16 tungsten in glass tetrodes (Thomas Recording, Fig. 1A) was inserted into the dorsal LGN, perpendicular to the brain surface, at the retinotopic locus corresponding to that of the intracellular recording in L4. A drifting grating was presented while each tetrode was lowered until spiking activity characteristic of LGN could be heard, usually between 11,000 and 13,000 $\mu \mathrm{m}$. All recordings were from lamina A (contralateral eye). No electrical or visual stimuli were presented that would allow the distinction of LGN $X$ and $Y$ cells.

To increase the likelihood of finding monosynaptically connected pairs, the V1 and LGN recording electrodes were positioned so as to obtain overlapping RFs. Typically, the LGN RFs were mapped first to obtain an estimate of their projections in $\mathrm{V} 1 \mathrm{using}$ published anatomical projection maps (Sanderson, 1971). All of the tetrodes were moved together if the LGN RFs were estimated to map $>5^{\circ}$ from the right area centralis.

Spike clustering. Spike waveforms from each tetrode in the LGN were clustered into individual units (Fig. 1D), online and offline as necessary, using a mixture of algorithmic and manual sorting (SpikeSort3D, Neuralynx). All clusters with spikes in the $0-1 \mathrm{~ms}$ bin (the estimated refractory period) of the interspike interval histogram were strictly rejected (Fig. 1D). Clusters whose corresponding RF estimate did not resemble a center-surround structure were excluded from further analysis.

Visual stimulation. The corneas were protected with neutral contact lenses after dilation of the pupil with atropoine solution and retraction of the nictitating membrane with phenylephrine $\mathrm{HCl}$. Spectacle lenses were chosen by the tapetal reflection technique to optimize the focus of stimuli on the retina, and the screen coordinates of the area centrali were recorded. Stimuli were presented on an Image Systems model M09 LV monochrome monitor operating at 125 frames per second, a spatial resolution of $1024 \times 786$ pixels, and a mean luminance of $47 \mathrm{~cd} / \mathrm{m}^{2}$. Computer-assisted hand-plotting routines were used with every cortical cell to provide initial estimates of critical parameters, including the location and size of the RF, optimal orientation, and optimal spatial and temporal frequency.

$R F$ mapping. RF structure was estimated by forward averaging of $V_{\mathrm{m}}$ and reverse averaging of spikes with several thousand frames of dense low-pass filtered white noise. Spikes were removed from the $V_{\mathrm{m}}$ using an automated algorithm before averaging. The noise was $16 \times 16^{\text {"RF pix- }}$ els" in space, covering $3^{\circ} \times 3^{\circ}$ to $5^{\circ} \times 5^{\circ}$, with frame duration of 16 or 24 ms (Fig. 1B). Each RF pixel could take one of four contrast values, ranging from black to white. The resulting estimate of the 3D spatiotemporal $\mathrm{RF}$ was displayed as the average white correlation minus the black correlation in frames of $5 \mathrm{~ms}$, out to $200 \mathrm{~ms}$ delay.

Both LGN and V1 cells have RFs that change over time, so the spatial $\mathrm{RF}$ is often defined at a particular point in time. We defined the spatial RF using the following procedure: The frame containing the pixel that produced the largest positive or negative response was found. The spatial RF was defined as the average of this frame with the one before and after. Then using the spatial RF, contours were defined at $20 \%$ of the maximum response value. We chose the $20 \%$ contour to be consistent with previous literature. An automated algorithm was used to remove spurious small contours outside of the main LGN center and 1-3 cortical RF subregions. For V1 RFs, separate ON and OFF contours were calculated. The maximum response and its negative were used to define contours for both the $\mathrm{ON}$ and OFF subregions. In this way, the spatial RF map contains information about the relative subregion strength, with weaker subregions displayed as smaller (Fig. 1C).

Spike-triggered averaging (STA) to reveal EPSPs. STAs were calculated by averaging $\pm 100 \mathrm{~ms}$ windows of cortical $V_{\mathrm{m}}$ centered on each LGN spike (Fig. 2B, STA). To correct for common stimulus modulation independent of a monosynaptic connection, a temporal jitter was applied to LGN spike times and the STA was recalculated (Fig. $2 B$, jittered STA). The amount of jitter was sampled from a uniform distribution matching the frame rate of the stimulus ( 16 or $24 \mathrm{~ms}$ ), a time scale that preserves the slower modulation of the $V_{\mathrm{m}}$ caused by the visual stimulus but eliminates the faster monosynaptic EPSP. By subtracting the jittered STA from the STA, we obtain the jitter-corrected STA, which reveals the mean EPSP triggered by the spikes of the presynaptic LGN cell (Fig. 2B, corrected STA)

Bootstrap significance testing of EPSPs. We developed a test of statistical significance to apply to the jitter corrected STAs to distinguish small EPSPs from background noise. For each LGN-V1 cell pair, the STA pro- 
A

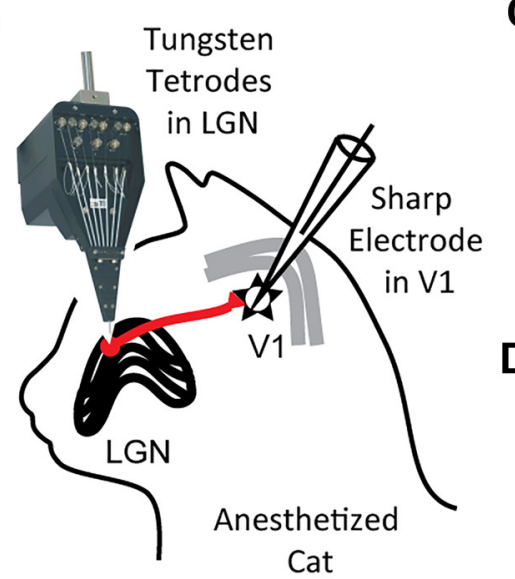

B
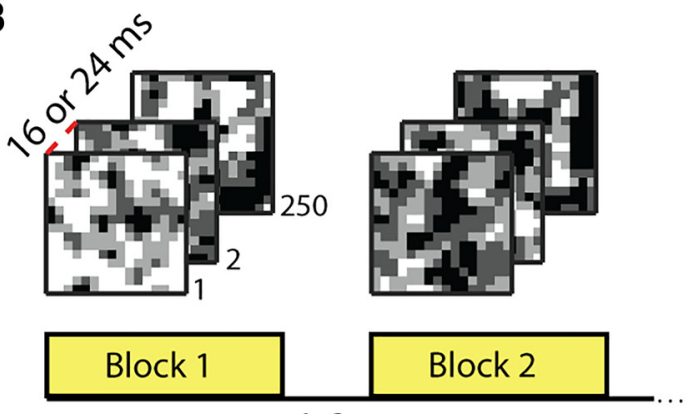

$1-2 \mathrm{~s}$

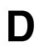

C Intracellular recording from V1
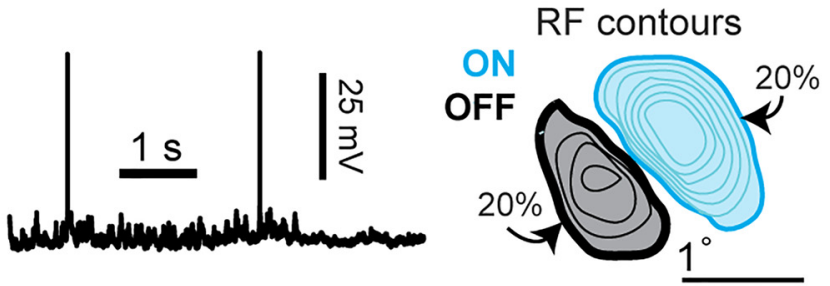

Extracellular recording from 1 Tetrode in LGN

Spike feature clusters Spike waveforms

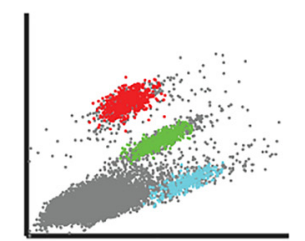

Inter-spike interval

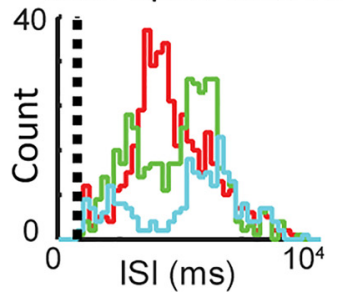

RF contours

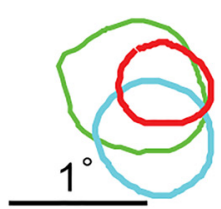

Figure 1. Paired recordings of LGN and V1 in the anesthetized cat brain. $\boldsymbol{A}$, An array of tungsten tetrodes was used to record the extracellular potentials of a population of $L G N$ cells, and a sharp electrode was used to measure intracellularly from a single V1 cortical cell in L4/6. B, Blocks of the 2D white-noise used to map LGN-V1 RFs. C, Sample recording of $V_{m}$ from a cortical V1 cell, along with the contours of the cell's RF, overlaid with the filled 20\% contour. Light blue represents subregions of the RF that prefer bright contrasts (ON). Black represents dark-preferring (OFF) subregions. The ON subregion is stronger, and therefore is larger and has more contour lines (20\%- $80 \%$ of the strongest pixel found in RF, in steps of $10 \%)$. The weaker OFF subregion is smaller and only has 20\%-50\% contour lines. D, Sample extracellular recordings of three LGN cells on one tetrode. Top, Spike features used to cluster spikes into different cells, along with each cell's spike waveform. Bottom, Interspike interval histogram for each cell, where the dashed line indicates the refractory period of $1 \mathrm{~ms}$. Contours of each LGN cell's RF center shown on the right.

duces a single mean and $\mathrm{SD}$ at each time point. To estimate the $\mathrm{SE}$ of this mean, we use bootstrap resampling.

First, jitter-corrected STAs with a positive deflection occurring within the monosynaptic latency range of $1.0-4.5 \mathrm{~ms}$ (Reid and Alonso, 1995) were selected for further analysis. Second, for each selected STA produced from $n$ spikes, a random sample of size $n$ was drawn with replacement to produce one bootstrap sample, and this process was repeated to generate 10,000 different bootstrap samples, each of size $n$. The distribution of the amplitude of the EPSP in each bootstrap sample was used to calculate the SEM EPSP amplitude, shown in Figure 3. In addition, this family of 10,000 bootstrap samples was used to estimate the mean and SE of the STA before and after LGN spikes. The STA after LGN spikes (Fig. $2 D$, top left, post-STA) is an estimate of the fluctuations produced by EPSPs from the presynaptic LGN cell, whereas the STA before LGN spikes (pre-STA) is an estimate of $V_{\mathrm{m}}$ noise independent of EPSPs. To establish significance of the EPSP, the bootstrap mean/SE of the postSTA was compared with that of the pre-STA. The pre-STA was subtracted from the post-STA (Fig. 2D, bottom left) and the $95 \%$ CI was calculated at each time point. If the CIs between 1.0 and $4.5 \mathrm{~ms}$ included at least 10 consecutive points above the zero line, the post-STA was classified as containing an EPSP, and otherwise was rejected as noise. Figure $2 D$ shows an example of a cell pair with EPSP that passed this significance test (left) and one that was rejected (right). This method excluded 5 potential EPSPs from our analysis and successfully rejected all 255 STAs without a positive deflection.

Fitting EPSPs. We fit each EPSP to a function of two exponentials as follows:

$$
V m(t)=c\left(1-e^{\frac{-t}{\tau_{\text {rise }}}}\right)^{5} e^{\frac{-t}{\tau_{\text {decay }}}}
$$

where $c$ is the amplitude of the EPSP, $\tau_{\text {rise }}$ is the time constant of the rising phase, and, $\tau_{\text {decay }}$ is the time constant of the decaying phase of the EPSP. We chose this function based on the best fit to data achieved using a variety of exponential, $\alpha$, and $\sigma$ functions.

Calculating cross-correlograms. Cross-correlograms were calculated between LGN and cortical spikes, for lags of $\pm 100 \mathrm{~ms}$, using $1 \mathrm{~ms}$ bins. LGN spikes were jittered to produce a jittered cross-correlogram. The amount of jitter was sampled from a uniform distribution matching the frame rate of the stimulus ( 16 or $24 \mathrm{~ms}$ ). The average of 100 such jittered cross-correlograms was used to represent the stimulus driven correlations between LGN and V1. The averaged jittered cross-correlogram (Fig. 4C, red traces) was subtracted from the cross-correlogram (Fig. 4C, black traces) to produce the corrected cross-correlogram (Fig. 4B). A significance threshold for the peak was set at 2.8 SDs above the baseline mean, where the baseline mean was defined as the average corrected cross-correlogram for lags of $-100:-20 \mathrm{~ms}$ and 20:100 ms. Cell pairs whose corrected cross-correlograms had a peak between 1 and $5 \mathrm{~ms}$ that exceeded the significance threshold were considered monosynaptically connected.

$R F$ similarity and overlap. Spatial similarity of LGN-V1 RFs was quantified using the RF overlap index (RFOI), the total intersected area between the $20 \%$ contour of the LGN and the $20 \%$ contour of the cortical spatial RFs, normalized by the area of the $20 \%$ LGN contour as follows:

$$
R F O I=\frac{\begin{array}{c}
\operatorname{area}(O N / O F F L G N \cap \operatorname{area}(O N / O F F \text { Cortex }) \\
-\operatorname{area}(\text { ON/OFF } L G N \cap \operatorname{area}(\text { OFF/ON Cortex })
\end{array}}{\operatorname{area}(L G N)}
$$


A
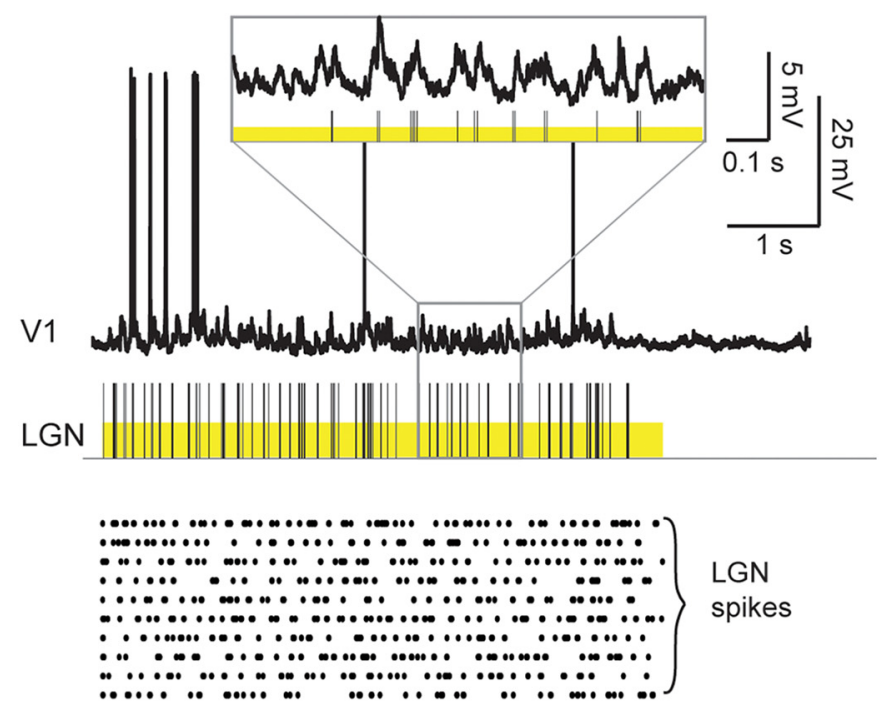

B

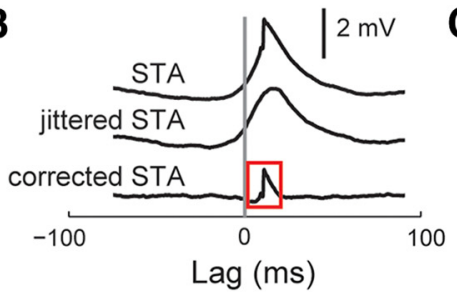

D

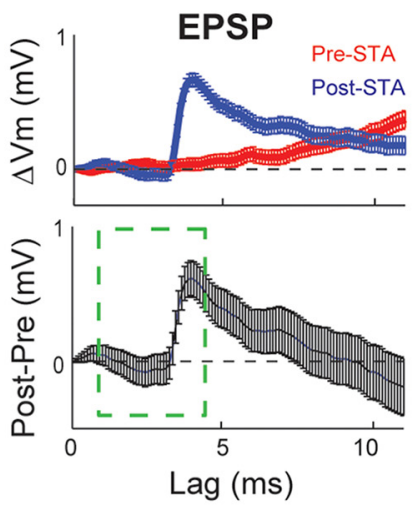

C

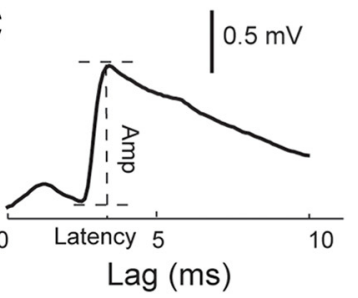

not an EPSP
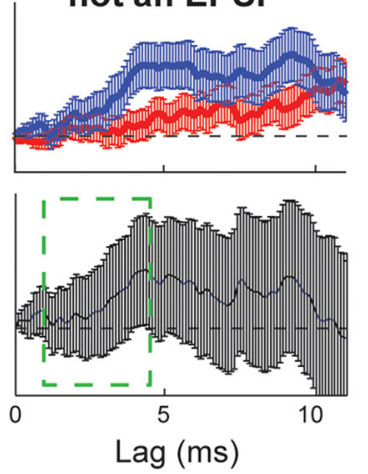

Figure 2. Detecting monosynaptic EPSPs in vivo. A, Simultaneous intracellular recording of a V1 (L4) neuron and extracellular recording of a connected LGN neuron in response to white noise (yellow bars). Raster plot shows LGN response to additional nonrepeating stimulus blocks. B, LGN STA of V1 $V_{m}$ (top), jittered STA used to remove stimulus induced correlations (middle), and difference revealing monosynaptic EPSP (bottom). "Lag" is time since LGN spike. C, Close-up of EPSP in B. D, Bootstrap significance test of EPSPs, for a sample LGN-V1 pair with an EPSP that passed (left column) and for a pair with an EPSP that failed (right column) the test. Top row, Mean and SEM of 10,000 bootstrapped estimates of the corrected STA for $10 \mathrm{~ms}$ before LGN spikes (pre-STA, red), representing $V_{m}$ noise, and after (post-STA, blue), representing contribution of LGN. Bottom row, Difference of post- and pre-STA. Error bars indicate SEM. Ten continuous points above zero, between 1 and $4.5 \mathrm{~ms}$ (green dashed window), indicate a significant EPSP (see Materials and Methods).
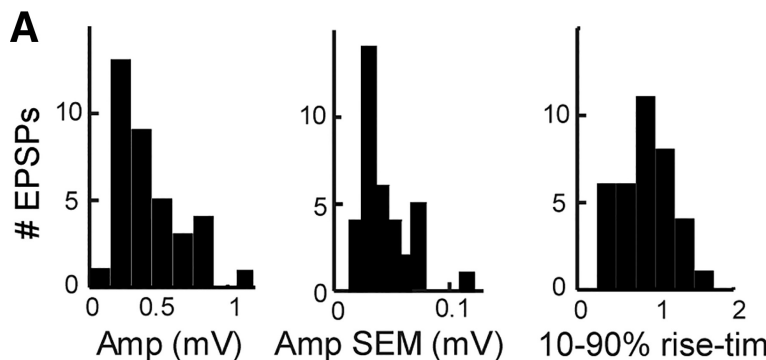

B
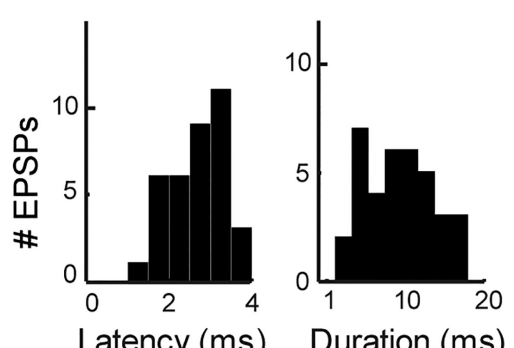

$10-90 \%$ rise-time
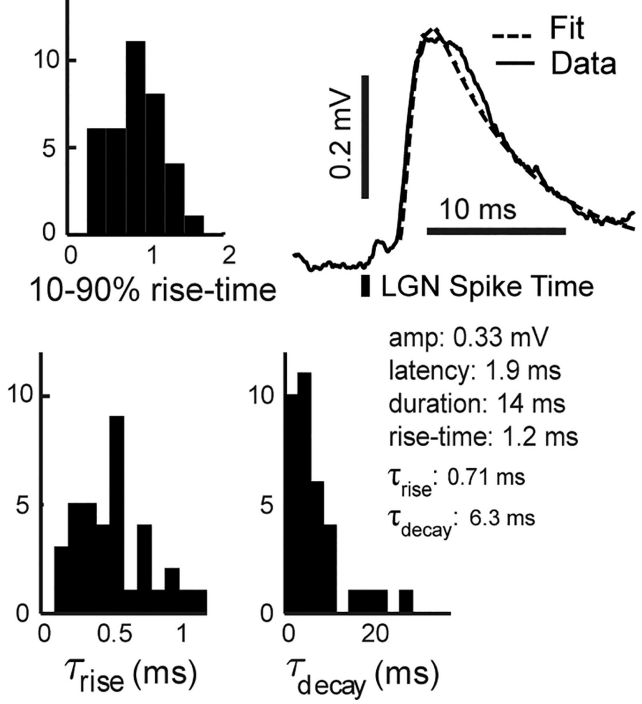

Figure 3. Distribution of EPSP features. $A$, Features extracted from mean EPSPs. Duration, $\tau_{\text {rise }}$ and $\tau_{\text {decay }}$ were calculated based on fits of the EPSP to a function of two exponentials (see Materials and Methods). SEM of the amplitude was calculated using bootstrapped STAs (see Materials and Methods). B, A sample EPSP and its fit.

Overlap of RF subregions of the same sign (ON-ON, OFF-OFF) contribute positively to the total intersected area and those of opposite sign contribute negatively. The RFOI ranges from 1, for perfect overlap of spatially identical RFs of the same sign, to -1 for perfect overlap of opposite sign (Fig. $5 C_{2}, x$-axis), and represents how much of the LGN RF center is contained within the subregion of the same or opposite sign of the V1 cell. Cells with no RF overlap are marked "N.O." to avoid confusion with an overlap of 0 arising from LGN cells perfectly straddling V1 subregions of opposite sign.
We also used a second RF overlap metric when calculating the correlation between various LGN-V1 response features and EPSP amplitude (Fig. $5 F$ ). This index, labeled $\mathrm{RFOI}_{2}$, is calculated as the total sign-matched intersected area between the LGN and RF contours, normalized by the square-root of the product of the LGN and V1 contour areas, and ranges from -1 to 1 . Because LGN RFs are smaller than V1 RFs, RFOI 2 does not reach its maximum value of 1 . In contrast, RFOI saturates at 1 (Fig. 5E). The two overlap indices are positively correlated. We focus on RFOI within the manuscript because it is a more intuitive metric, but added $\mathrm{RFOI}_{2}$ to make sure saturation of the index did not bias the correlation of RF overlap with EPSP amplitude (Fig. 5F).

Response correlation. To capture the similarity in the overall neuronal response of LGN-V1 pairs, we defined an LGN-V1 response correlation metric. The response correlation was calculated as follows: The cortical $V_{\mathrm{m}}$ was demeaned to allow comparison of response correlation between cells with different resting potentials. LGN spikes were jittered to produce a jittered STA of the cortical $V_{\mathrm{m}}$, for lags of -20 to $20 \mathrm{~ms}$. We chose this window because it captured the peak of the stimulus-driven response correlations, as opposed to baseline correlations at larger lags. As before, the jitter was sampled from a uniform distribution matching the frame rate of the stimulus. For connected pairs, the EPSP was not visible in the jittered STA (Fig. $2 B$ ). We averaged the jittered STA to obtain a scalar, representing the LGN-V1 response correlation. Response correlation is high for a cell pair when LGN spikes arrive in proximity to depolarization of the cortical $V_{\mathrm{m}}$, and low when LGN spikes arrive in proximity to $V_{\mathrm{m}}$ hyperpolarization. Cell pairs with 
large RFOI generally had high response correlation, and those with low RFOI generally had low response correlation (Fig. 5E).

$R F$ distance. Prior work (Alonso et al., 2001) has shown that the probability of connection is a function of the distance between LGN centers and peak of the overlapping V1 subregion. As another metric of LGN-V1 RF similarity, we calculated the distance between the strongest pixel in the LGN RF and the strongest pixel in the sign-matched V1 RF subregion. If no signmatched V1 subregion existed, no distance was calculated for that cell pair. As in Alonso et al. (2001), we calculated the distance along the width and height axes of the V1 subregion, by performing a coordinate transformation based on the orientation angle of the V1 RF.

Orientation tuning. We used drifting sinusoidal gratings of 16 different orientations from $0^{\circ}$ to $360^{\circ}$. Gratings were optimized for the V1 cell with spatial frequency typically in the range of $0.5-1$ cycles/degree and temporal frequency of $2-2.5$ cycles/s. The response of a cell to a grating at angle $\theta_{\mathrm{k}}$ can be represented by the complex vector $\mathrm{R}_{k} e^{\left(\mathrm{i} 2 \theta_{k}\right)}$, where the angle doubling serves to equalize gratings of the same orientation that drift in opposite directions $\left(180^{\circ}\right.$ apart). An orientation bias vector can then be calculated as the sum of response vectors divided by the sum of the response magnitudes across all orientations. The magnitude of this complex bias vector represents the orientation selectivity index (OSI), and the phase is the preferred angle $\left(\theta_{\text {pref }}\right)$ of the neuron as follows:

$$
\text { OSI }=\left|\frac{\sum_{k} \mathrm{R}_{k} e^{\left(\mathrm{i} 2 \theta_{k}\right)}}{\sum_{k} \mathrm{R}_{k}}\right| \quad \theta_{\text {pref }}=\text { phase }\left(\frac{\sum_{k} \mathrm{R}_{k} e^{\left(\mathrm{i} 2 \theta_{k}\right)}}{\sum_{k} \mathrm{R}_{k}}\right)
$$

The OSI is bounded between 0 and 1, with higher values indicating increased selectivity for orientation. This measure of OSI is equivalent to 1 - circular variance (Ringach et al., 1997).

\section{Results}

Our goal was to characterize the properties of EPSPs generated in vivo by LGN neurons in their target V1 neuron. To this end, we recorded the intracellular potential $\left(V_{\mathrm{m}}\right)$ of a $\mathrm{V} 1$ neuron and simultaneously recorded the extracellular spikes from groups of LGN neurons, in response to a 2D dense white noise stimulus, in the anesthetized cat (Fig. 1).

\section{Identifying monosynaptic EPSPs}

Each spike from a monosynaptically connected LGN cell will produce a (single-spike) EPSP in the postsynaptic cortical $V_{\mathrm{m}}$. Because each single-spike EPSP is small and the $V_{\mathrm{m}}$ is noisy, hundreds of single-spike EPSPs must be averaged to reveal the mean EPSP (Fig. $2 A-C$ ), a procedure known as spike-triggered average (STA). To identify monosynaptic connections, we generated STAs of the cortical $V_{\mathrm{m}}$ for all simultaneously recorded LGN cells. The STA (Fig. $2 B$, top) of a connected LGN-V1 pair contains both the LGN-driven EPSP and a slower stimulus-driven component. We isolated and removed the stimulus-driven component (Fig. 2B, middle) by generating a new STA with jittered spike times and subtracting it from the STA. The corrected STA (Fig. 2B, bottom) for connected LGN-V1 pairs contains an EPSP (Fig. 2C).

Given the small size of the EPSPs, we developed a bootstrap procedure (see Materials and Methods) to determine the significance of each putative EPSP in the corrected STA. Briefly, we gen- erated bootstrap resamples of the corrected STA to estimate its mean and SE. We subtracted the mean for the $10 \mathrm{~ms}$ preceding LGN spikes (which represents baseline noise) from the mean for the $10 \mathrm{~ms}$ following spikes and used 95\% CIs calculated from the bootstrap samples to establish a threshold for EPSP detection (Fig. 2D).

\section{Properties of monosynaptic thalamocortical EPSPs}

From the dataset produced by a total of 44 experiments, which included $44 \mathrm{~V} 1$ cortical cells and 543 LGN cells, we identified 36 monosynaptic EPSPs (Table 1). We classified V1 neurons as either simple $(n=24)$ or complex $(n=20)$ based on their RF structure (Hubel and Wiesel, 1962; Jones and Palmer, 1987) and the degree of firing rate modulation to drifting gratings (Skottun et al., 1991). We found at least one LGN connection onto 23 of the simple cells, but we did not detect any connections onto any of the complex cells. Experiments in which no connected pairs were found were excluded from further analysis, resulting in a dataset of 23 cortical cells and 290 LGN cells (Table 2).

Some simple cells received input from more than one of the simultaneously recorded LGN cells, producing 36 LGN connections onto $23 \mathrm{~V} 1$ cortical neurons (Table 2). We found connections onto simple cells in both L4 (500-950 $\mu \mathrm{m})$ and L6 (>1200 $\mu \mathrm{m})$. EPSPs from L4 and L6 had no indistinguishable features and were analyzed together.

The distribution of EPSP amplitude across the 36 connections was not Gaussian (Fig. 3A). The mean EPSP amplitude was $0.42 \pm 0.26 \mathrm{mV}( \pm \mathrm{SD})$, and the median was $0.32 \pm 0.34 \mathrm{mV}$ ( \pm interquartile range). The SEM of each EPSP amplitude was calculated using 10,000 bootstrapped samples (see Materials and Methods), with a mean of $0.044 \pm 0.021 \mathrm{mV}$. The mean latency to onset after LGN spike was $2.6 \pm 0.64 \mathrm{~ms}$, with a median of $2.7 \pm$ $1.1 \mathrm{~ms}$. The mean $10 \%-90 \%$ rise time was $1.1 \pm 0.74 \mathrm{~ms}$. To obtain population measures of kinetic parameters of the EPSPs, we fit each EPSP to a function of two exponentials (see Materials and Methods) and obtained time constants for the rise $\left(\tau_{\text {rise }}\right)$ and 
A
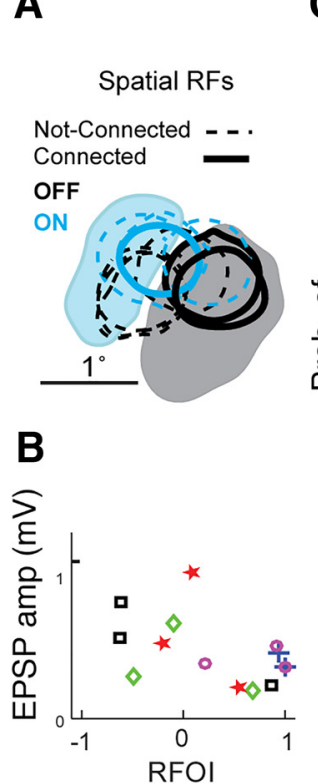

$\mathbf{C}_{1}$
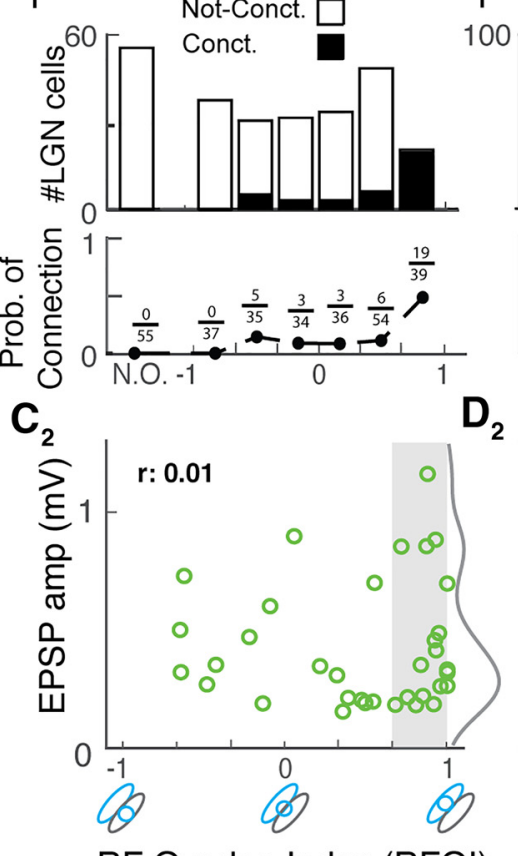

RF Overlap Index (RFOI)
$D_{1}$

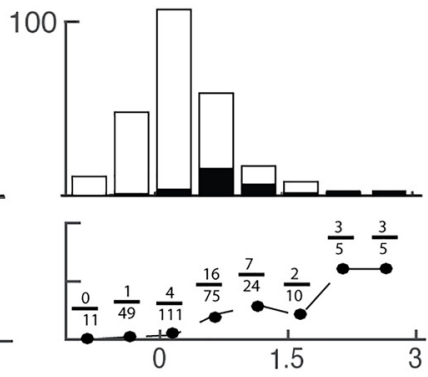

$\mathrm{D}_{2}$

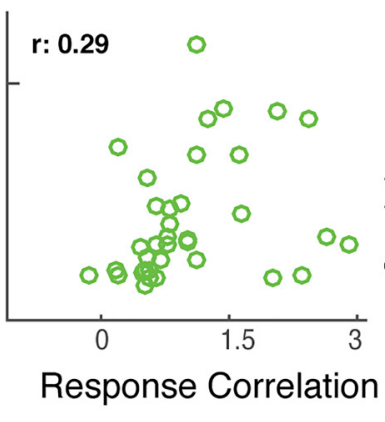

E

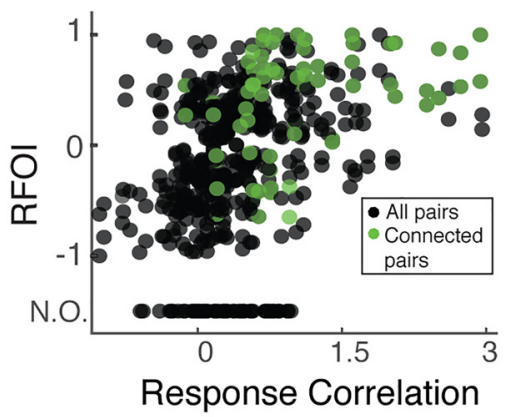

F Correlation with EPSP amp

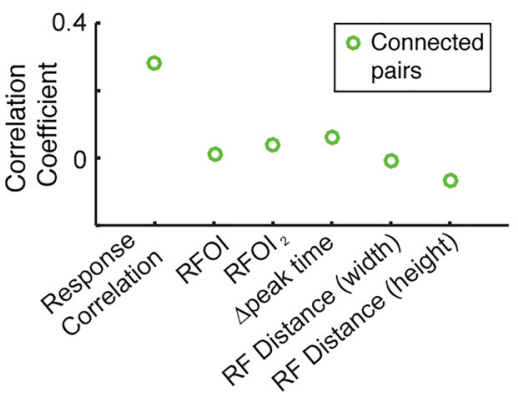

Figure 5. Correlation of LGN-V1 response properties with probability of connection and EPSP amplitude. A, Filled spatial RF contours (20\%) of a sample L4 V1 cell with $20 \%$ contours of 10 simultaneously recorded LGN cells with the three connected shown in bold lines. Blue represents ON RF subregions. Gray/black represents OFF subregions. RFOl is quantified as the amount of sign-matched overlap between LGN and V1 RF contours (see Materials and Methods, $x$-axis of $C_{2}$ ). $B$, EPSP amplitude versus RFOI for 5 cortical cells with 3 LGN connections onto each cell. Three connected cells in $\boldsymbol{A}$ are shown in purple circles. C, Relationship between RFOl and probability of connection (1) and EPSP amplitude (2). $\boldsymbol{C}_{\boldsymbol{1}}$, Histograms are overlaid on top of each other and show the RFOI distribution of connected and not-connected LGN-V1 pairs. Pairs with no spatial RF overlap are marked "N.0." to disambiguate from an RF0I of 0 . Fractions are number of connected pairs divided by number of all recorded pairs in each RFOI bin. $C_{2}$, Gray curve indicates the smoothed histogram of EPSP amplitude for connections with RFOI $>0.67$. $\boldsymbol{D}$, Same as $\boldsymbol{C}$, but for response correlation. $\boldsymbol{E}$, Relationship between RFOI and response correlation. $\boldsymbol{F}$, Pearson correlation coefficient $(r)$ for correlation of various $L G N-V 1$ response features and EPSP amplitude.

Table 1. Paired recording experiment cell counts ${ }^{a}$

\begin{tabular}{llclc}
\hline $\begin{array}{l}\text { Cortical } \\
\text { cell type }\end{array}$ & $\begin{array}{l}\text { Recorded } \\
\text { cortex }\end{array}$ & $\begin{array}{l}\text { Connected } \\
\text { cortex }\end{array}$ & $\begin{array}{l}\text { Recorded } \\
\text { LGN }\end{array}$ & $\begin{array}{l}\text { Connected } \\
\text { LGN }\end{array}$ \\
\hline Complex & 20 & 0 & 253 & 0 \\
Simple & 24 & 23 & 296 & 36
\end{tabular}

$\overline{{ }^{a} \text { Number of recorded and connected V1 and LGN neurons across } 44 \text { paired recording experiments, separated by V1 }}$ functional cell type.

decay phases $\left(\tau_{\text {decay }}\right)$ as well as an estimate of the duration of the EPSP (Fig. $3 B$ ). The mean $\tau_{\text {rise }}$ was $0.51 \pm 0.26 \mathrm{~ms}$ with a median of $0.53 \pm 0.33 \mathrm{~ms}$. The mean $\tau_{\text {decay }}$ was $7.9 \pm 8.4 \mathrm{~ms}$ with a median of $5.2 \pm 6.0 \mathrm{~ms}$, and the mean EPSP duration was $9.7 \pm$ $4.2 \mathrm{~ms}$.

The EPSP amplitudes in our in vivo dataset are notably smaller than those measured in a previous in vitro study of the cat thalamocortical synapse (Stratford et al., 1996), and closer to in vivo measurements of thalamocortical EPSPs in the rat barrel cortex (Bruno and Sakmann, 2006). Consideration of LGN spike synchrony renders our average LGN to V1 EPSPs even smaller than the amplitudes reported here (see Discussion).

\section{Relationship between LGN-V1 spike cross-correlogram and STA}

In principle, our intracellular recordings should allow us to detect synaptic inputs using both the LGN spike-triggered-average of the V1 $V_{\mathrm{m}}$ and the cross-correlogram of LGN and V1 spikes (Alonso et al., 1996, 2001). However, detecting a brief monosynaptic peak in a cross-correlogram requires several thousand spikes, which we usually did not have due to the typically shorter duration of intra versus extracellular recordings (our range of LGN spikes $=122$ to 2905). Therefore, with the exception of four connected pairs (one of which is shown in Fig. 4), we were not able to detect a significant monosynaptic peak (see Materials and Methods) in LGN-V1 cross-correlograms. This suggests that the STA is a more sensitive method than the spike cross-correlogram for detection of synaptic connections in vivo.

The four LGN cells with significant cross-correlogram peaks all exhibited relatively high firing rates. One of the four cells was connected to a cortical cell with two additional detected LGN inputs in our dataset. The three LGN inputs converging onto the same cortical cell had similar EPSP amplitudes and RF overlaps (Fig. 4A) but different firing rates in response to white noise stimuli (A: 13.5 spikes/s, B: 4.3 spikes/s, C: 5.1 spikes/s, cortical firing rate 5.7 spikes/s). We found a significant peak in the crosscorrelogram of only one of three LGN cells (cell A, Fig. 4B), the cell with the highest firing rate. It is also possible that cell $\mathrm{A}$ received a "boost" from cell B, which on average fired before cell A according to pairwise cross-correlograms among the three LGN inputs (Fig. 4C). The spikes of cell B, which arrive first, would depolarize the cortical $V_{\mathrm{m}}$, leading to higher likelihood of cell A's spikes to produce a cortical spike and a significant peak in the cross-correlogram between cell A and cortex. This observation is consistent with prior reports of increased efficacy of a second LGN spike arriving within a $\sim 10 \mathrm{~ms}$ window of a prior spike (Usrey et al., 2000), as well as with the $\sim 10$ ms time constant of cell membranes in V1. 
Table 2. Features of connected pairs ${ }^{a}$

\begin{tabular}{|c|c|c|c|c|}
\hline $\begin{array}{l}\text { Cortical } \\
\text { cell no. }\end{array}$ & $\begin{array}{l}\text { Cell } \\
\text { type }\end{array}$ & $\begin{array}{l}\text { Cortical } \\
\text { layer }\end{array}$ & $\begin{array}{l}\text { Recorded } \\
\text { LGN cells }\end{array}$ & $\begin{array}{l}\text { Connected } \\
\text { LGN cells }\end{array}$ \\
\hline 1 & RS & L6 & 4 & 1 \\
\hline 2 & RS & L4 & 22 & 3 \\
\hline 3 & RS & L4 & 7 & 3 \\
\hline 4 & RS & L4 & 6 & 1 \\
\hline 5 & FS & L4 & 18 & 2 \\
\hline 6 & FS & L4 & 12 & 3 \\
\hline 7 & RS & L4 & 12 & 2 \\
\hline 8 & RS & L4 & 10 & 3 \\
\hline 9 & RS & L4 & 11 & 1 \\
\hline 10 & RS & L6 & 11 & 1 \\
\hline 11 & RS & L4 & 19 & 1 \\
\hline 12 & RS & L4 & 14 & 1 \\
\hline 13 & FS & L4 & 8 & 1 \\
\hline 14 & FS & L4 & 21 & 1 \\
\hline 15 & RS & L4 & 11 & 1 \\
\hline 16 & RS & L6 & 15 & 1 \\
\hline 17 & FS & L6 & 25 & 3 \\
\hline 18 & RS & L4 & 8 & 1 \\
\hline 19 & RS & L4 & 14 & 1 \\
\hline 20 & RS & L4 & 9 & 1 \\
\hline 21 & FS & L4 & 14 & 1 \\
\hline 22 & RS & L6 & 13 & 2 \\
\hline 23 & FS & L4 & 6 & 1 \\
\hline Total 23 & & & 290 & 36 \\
\hline
\end{tabular}

${ }^{a}$ Electrophysiological cell type and cortical layer of $23 \mathrm{~V} 1$ simple cells that were connected to at least one simultaneously recorded LGN cell. Numbers of recorded and connected LGN cells are also given.

We conclude that the cross-correlogram peak is influenced by the strength of the synaptic connection, the presynaptic firing rate, as well as the temporal correlations among the presynaptic population (Usrey et al., 2000). In contrast, the STA is a more sensitive method that is influenced mainly by synaptic strength and not by presynaptic firing rate. We note that both methods will fail to detect two perfectly synchronized LGN inputs, rendering them as one larger input. However, as detailed in Discussion, such synchrony is infrequent in the LGN.

\section{LGN-V1 response similarity is strongly correlated with the likelihood of monosynaptic connections}

Simple cell responses are characterized by one or more elongated ON (driven by bright-contrast stimuli) or OFF (driven by darkcontrast stimuli) RF subregions. In contrast, LGN neurons exhibit a roughly circular center-surround organization in which the center and the surround respond to opposite sign contrasts. Hubel and Wiesel's feedforward model (Hubel and Wiesel, 1962) predicts that V1 cells receive inputs from LGN cells with similar response properties, in other words, LGN cells whose RFs overlap and match in sign with the V1 RF. Seminal extracellular studies have lent support to this hypothesis, showing that spatial and temporal similarity between LGN and V1 RFs is highly correlated with the probability of a positive peak in the spike cross-correlogram (Tanaka, 1983; Reid and Alonso, 1995; Alonso et al., 2001). However, as discussed above, STA of intracellular measurements is a more sensitive detector of monosynaptic connections compared with the cross-correlogram. Therefore, connections detected using the STA may reveal different rules of connectivity than the rules revealed by cross-correlogram analysis.

We quantified the similarity of the spatial RFs by calculating the degree of sign-matched overlap between the LGN and V1 RF contours using the RFOI (see Materials and Methods). RFOI was calculated as the area of intersection of the $20 \%$ contours of the cortical and LGN RFs, normalized by the area of the LGN RF contour. An RFOI of 1 indicates perfect overlap of the LGN RF with the sign-matched subregion of the cortical RF (Fig. $5 C_{2}$, $x$-axis). The RFOI takes into account the relative strength of V1 subregions because the $20 \%$ RF contour will be larger for the stronger subregion (see Materials and Methods). In Figure 5A, the larger OFF subregion contour of the cortical RF is stronger than the smaller ON subregion contour.

The distribution of RFOI is different between connected and not-connected LGN-V1 pairs (two-sample Kolmogorov-Smirnov [KS] test, $p=0.04)$. While the number of connected pairs increases at higher overlaps, not-connected pairs are distributed more uniformly (Fig. $5 C_{1}$ ). The probability of connection decays dramatically with reduction in overlap (Fig. $5 C_{1}$, bottom) and reaches its maximum of $49 \%$ for RFOIs between 0.67 and 1 . We found 8 of 36 connected LGN cells with negative RFOI values, arising from predominant overlap of the LGN RF center with the V1 RF subregion of opposite sign. We refer to these connections as "nonspecific." In addition, we recorded 55 pairs without RF overlap (Fig. $5 C_{1}$, N.O.), and none were connected. Thus, in agreement with extracellular studies, we find a steep increase in probability of geniculocortical connections as a function of RF overlap.

We recorded from 20 LGN cells with high RF overlap (RFOI between 0.67 and 1), which were not connected to the simultaneously recorded V1 cell. Because RFOI ignores the temporal dynamics of LGN and V1 responses, the possibility remained that differences in the temporal properties of these cells reduced the correlation of their response with the cortical cell and thereby reduced their connection probability. To test this hypothesis, we calculated the probability of connection as a function of response correlation between LGN-V1 pairs. Response correlation was quantified as the mean value of the jittered STA, which represents stimulus-induced correlations between LGN spikes and V1 $V_{\mathrm{m}}$ responses (see Materials and Methods). The range of response correlation values for all cell pairs was $\sim-1$ to $3 \mathrm{mV}$, and response correlation showed a positive correlation with RFOI as expected (Fig. 5E). The distribution of response correlation (Fig. $5 D_{1}$ ) was different between connected and not-connected LGN-V1 pairs (two-sample KS test, $p<0.001$ ), with the distribution for connected pairs shifted toward higher values.

The probability of connection was higher for larger response correlation (Fig. $5 \mathrm{D}_{2}$, bottom) and reached its maximum of 0.6 for the highest response correlation values. However, regardless of the bin size used, there were always not-connected pairs at the highest response correlation values, suggesting that response similarity does not guarantee synaptic coupling in the thalamocortical pathway.

Alonso et al. (2001) found that RF overlap increases, but does not guarantee, the probability of connections detected with spike cross-correlograms, and pointed to several other factors that increase connection probability. However, the possibility remained that a more sensitive detector may reveal that all cell pairs with a high degree of RF overlap and response similarity are weakly connected. Our data rule out this hypothesis and confirm the findings of Alonso et al. (2001). These findings illustrate the sparseness of the geniculocortical connectivity at the single-cell level, despite the massive number of converging geniculate axons per unit area (Freund et al., 1985; Humphrey et al., 1985; Peters and Payne, 1993; da Costa and Martin, 2011). 


\section{LGN-V1 response similarity is not correlated with EPSP amplitude}

Given that LGN-V1 RF and response similarity impacts the likelihood of synaptic connections, it is reasonable to hypothesize that it may also affect the amplitude of synaptic EPSPs. We expected that cell pairs with high response and RF similarity would exhibit the largest EPSP amplitudes. Surprisingly, we did not find a significant correlation between EPSP amplitude and RFOI across the population of connected pairs (Fig. $5 C_{2}, r=0.01, p=$ 0.94). We also did not find a correlation between EPSP amplitude and RFOI for LGN cells connected onto the same cortical cell (5 cortical cells with 3 connections each, Fig. $5 B$ ). This rules out the possibility that differences in the cortical cell's input resistance or mean $V_{\mathrm{m}}$ could act to wash out correlations between EPSP amplitude and RFOI. For the population, some of the largest EPSPs appear in the highest RFOI bin $\left(0.67-1\right.$, Fig. $\left.5 C_{2}\right)$, but this arises from the larger number of connected cells in this bin (19 of total 36 ). Figure $5 C_{2}$ (gray region) shows that, although the 20 cell pairs with the highest RFOI $(>0.67)$ exhibited both small and large EPSPs, the distribution was biased toward small values of $\sim 0.25 \mathrm{mV}$. We used a two-sample KS test to determine whether the distribution of EPSP values was significantly different between different RFOI bins, between connections with negative and positive RFOI, and between all RFOI bins and only the highest bin. We found no difference in these distributions, confirming the lack of a relationship between RF overlap and EPSP amplitude.

We also did not find a significant correlation between the EPSP amplitude and response correlation (Fig. $5 D_{2}, r=0.29, p=$ 0.38 ) of connected pairs. We considered several other measures of LGN-V1 response similarity and calculated their correlation with EPSP amplitude (Fig. 5F). We used a second measure of RF overlap $\left(\mathrm{RFOI}_{2}\right.$, see Materials and Methods; $\left.r=0.04, p=0.84\right)$, which did not saturate at the maximum value of 1 . We also considered the difference between the peak of the LGN-V1 RF time course ( $\Delta$ peak time; $r=0.07, p=0.66$ ), an indication of the temporal similarity of the two RFs. Finally, we considered the distance between the strongest pixel of the LGN and V1 spatial RFs (Alonso et al., 2001), along both the width $(r=-0.06, p=$ 0.73 ) and height $(r=-0.00, p=0.99)$ axes of the overlapping V1 subregion (see Materials and Methods). None of these features exhibited a significant correlation. Our results show that RF and response similarity of LGN-V1 pairs is not correlated with the amplitude of synaptic EPSPs. These findings contradict previous findings in the mouse cortex (Cossell et al., 2015), as well as expectations from the basic formulation of Hebbian plasticity (see Discussion).

\section{Properties of connections onto V1 RS and FS cells}

In vitro studies report significant differences in the number and amplitude of thalamocortical EPSPs onto RS, putatively excitatory, and FS putatively inhibitory, cortical cells (Cruikshank et al., 2007; Schiff and Reyes, 2012; Kloc and Maffei, 2014), suggesting an early divergence in the strength of cortical excitation and inhibition. To determine whether the properties of LGN-V1 connections depend on the electrophysiological class of the target V1 cell, we classified the 23 connected V1 cells into RS $(n=16)$ and FS $(n=7)$ types, based on their intracellularly measured electrophysiological properties (McCormick et al., 1985; Contreras and Palmer, 2003; Nowak et al., 2003). FS cells responded to current injection with nonaccommodating trains of high-frequency firing of up to $300 \mathrm{~Hz}$ (Fig. 6A), and had smaller spike widths $(<0.8$ $\mathrm{ms}$, measured at the base) with pronounced AHPs (Fig. 6B). In contrast, RS cells showed lower frequency firing, accommodating trains in response to current injection, longer duration action potentials $(>0.8 \mathrm{~ms}$ ), and absence of a clear AHP (Fig. $6 A, B)$.

The 16 RS V1 cells had a total of 24 LGN connections and the 7 FS V1 cells had a total of 12 connections (Table 2), producing similar average number of connections per RS $(1.5 \pm 0.82)$ and FS $(1.7 \pm 0.95)$ cell. Both the two-sample $t$ test $(p=0.56)$ and the two-sample KS test $(p=1)$ fail to reject the null hypothesis that RS and FS cells have equal average number of LGN connections, but larger datasets may reveal differences in the number of connections onto each cell type. More notably, the distribution of EPSP amplitudes was not different (two-sample KS test) between RS and FS cells (Fig. 6C, $y$-axis), and neither was the distribution of onset latency (Fig. 6C, $x$-axis). We also did not find any differences in other EPSP features of RS and FS cells. Our findings indicate that, in the cat visual system, the thalamocortical connection onto putatively excitatory and putatively inhibitory cells is of similar strength.

Aside from differences in synaptic strength, L4 RS and FS cells receive different patterns of thalamic input in the rabbit somatosensory cortex (Swadlow and Gusev, 2002; Alonso and Swadlow, 2005), with FS cells receiving nonspecific thalamic input, resulting in broadly tuned RFs. Nonspecific thalamocortical input in the visual system corresponds to input from LGN cells with signmismatched LGN-V1 RF overlap. Because nonspecific inputs specifically to FS, putatively inhibitory, cells may change the spatiotemporal properties of feedforward inhibition; we were interested in whether FS cells in V1 also received nonspecific thalamic inputs.

We designed our RF overlap index so that nonspecific LGN inputs would produce negative RFOI values (Fig. $5 C_{2}, x$-axis). As shown for the sample experiments in Figure 6F, LGN cells connected to V1 FS cells (right column) often showed only partial overlap with the sign-matched V1 subregion, indicating nonspecific connections $(\mathrm{RFOI}<0)$. In contrast, $\mathrm{LGN}$ input to RS cells exhibited specific (sign-matched RF overlap) connections, suggesting a target-cell specific difference in the RF similarity of LGN inputs.

Eight of the 36 connected LGN cells in our dataset provide nonspecific input to their target cortical cell (Fig. $6 D, E$ ). Of these, the majority ( 6 of 8 ) were connected to FS cells (Fig. $6 D_{1}$ ). These 6 cells comprised half of the 12 LGN cells connected to FS cells (Fig. $6 D_{2}$ ). In contrast, the majority of LGN cells with positive RFOI values (22 of 28 ) were connected to RS cells (Fig. $6 D_{1}$ ). Conversely, the majority of LGN connections onto RS cells ( 22 of 24) had positive RFOI values (Fig. $6 D_{2}$ ), indicating specific connections. The lack of nonspecific connections onto RS V1 cells does not appear to be caused by a sampling bias in our paired recordings. From a total of 56 LGN cells whose RFs exhibited nonspecific overlap with the simultaneously recorded RS V1 cell, only 2 were connected (Fig. $6 E$ ), whereas this ratio was 6 of 40 for FS cells. Our small dataset supports the notion that inhibitory neurons in the inputs layers of V1 receive less specific thalamic inputs compared with excitatory neurons, consistent with observations in rabbit somatosensory cortex (Swadlow and Gusev, 2002).

However, the consequences of this divergence in input specificity on the properties of the cortical RF are not clear. We examined the RF linearity, a metric used to describe the degree of "simpleness" of a V1 RF (Priebe et al., 2004), by calculating correlation between $\mathrm{ON}$ and $\mathrm{OFF}$ responses at the time of maximal response (Priebe et al., 2004). We found no correlation between 
A

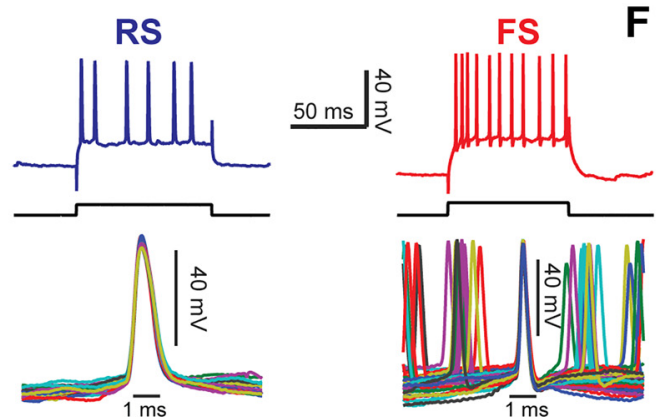

B

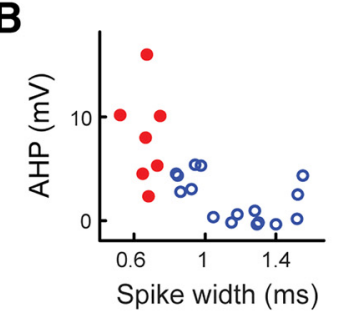

$D_{1}$

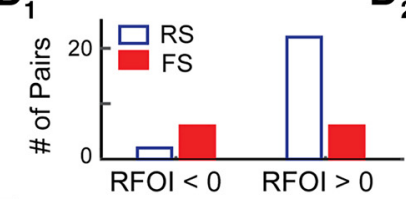

E

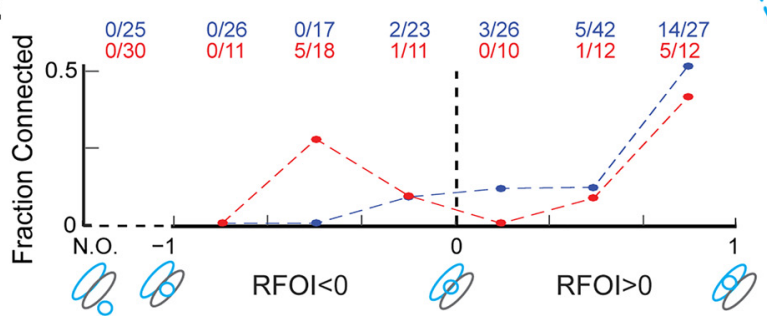

C

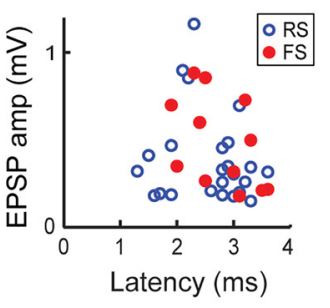

$D_{2}$

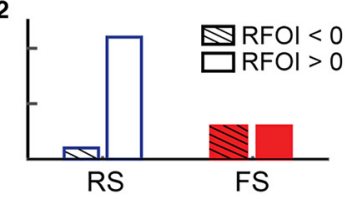

RS

FS
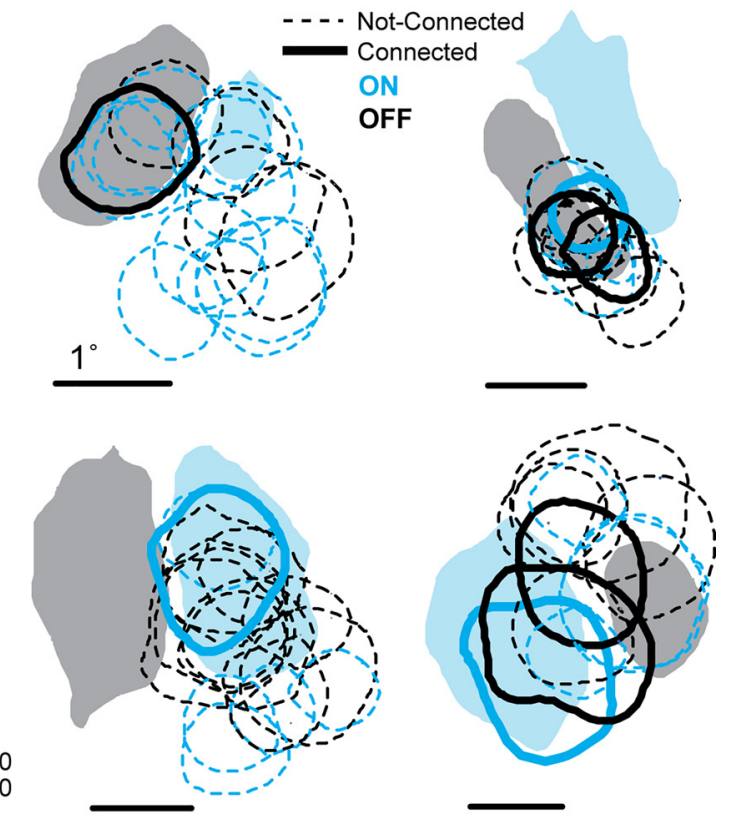

Not-Connected

ected

OFF
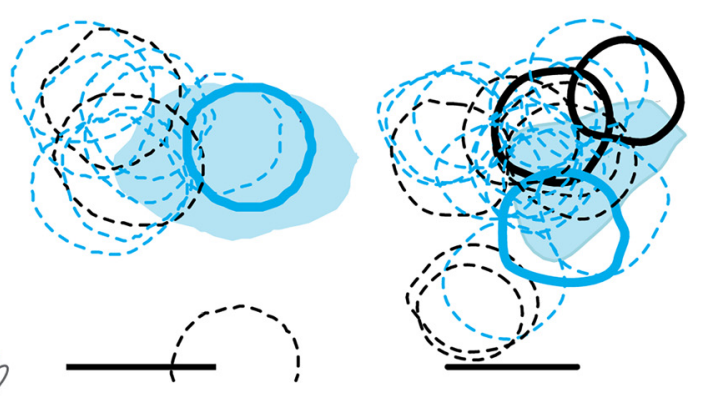

Figure 6. Properties of LGN connections onto V1 RS and FS cells. $A$, Sample RS and FS cells in L 4 V1. Top, Typical $V_{m}$ responses to depolarizing current injections. Bottom, Overlaid action potentials for each cell. B, Spike AHP and spike width at base were used, along with firing rate and response to current injection, to classify RS ( $n=16)$ and FS $(n=7)$ cells. C, EPSP amplitude plotted against EPSP latency for EPSPs in V1 RS (blue) and FS (red) cells. D, LGN-V1 RF overlap is different for connections onto RS and FS cells. Number of nonspecific (RFOI $<0$ ) and specific (RF0I $>0$ ) LGN connections onto V1 RS and FS cells, separated by RFOI (1) and by V1 cell type (2). $\boldsymbol{E}$, Fraction of connected LGN cells, separated by cell type of target V1 cell, for each RF0l bin. Raw numbers, pooled across all experiments, are shown above each bin. $\boldsymbol{F}$, Filled $20 \%$ RF contours of a single V1 cell overlaid with circular RF contours of simultaneously recorded LGN cells, with the connected LGN cells shown in bold, for six different experiments. Calibration: $1^{\circ}$. Blue represents $0 \mathrm{~N}$ subregions of the RF. Gray/black represents $0 \mathrm{FF}$ subregions. The V1 cells in the left column are RS, the right column FS.

this parameter and either RS/FS cell type or LGN input specificity as measured by RFOI. Furthermore, previous studies from our laboratory have shown similar orientation tuning bandwidth for V1 RS and FS cells (Cardin et al., 2007). Nonetheless, we report our observations here so that future studies may contradict or corroborate them.

\section{V1 orientation preference is not inherited from single LGN inputs}

It is generally assumed that orientation selectivity in the cat and primate first emerges in V1 from the convergence of LGN cells with spatially aligned RFs. However, some debate remains on whether weak orientation bias of individual LGN cells (Suematsu et al., 2012) also contributes to orientation selectivity in V1 (Shou and Leventhal, 1989; Vidyasagar et al., 2015). In contrast to the situation in mice (Scholl et al., 2013; Sun et al., 2016; but see Kondo et al., 2016), there is little evidence for the transfer of orientation bias from single LGN cells to V1 in cats (Vidyasagar et al., 2015) or monkeys. If such were the case, the orientation bias of LGN cells, when present, should match or approximate the optimal orientation of their target V1 neurons. We quantified the orientation selectivity of simultaneously recorded LGN and V1 cells for a subset of the experiments (see Materials and Methods). We used $V_{\mathrm{m}}$, instead of spikes, to calculate the orientation selectivity of the $\mathrm{V} 1$ cell, so we could more directly compare the effect of an LGN cell's orientation bias on its target $\mathrm{V} 1$ cell.

We calculated the OSI and the preferred orientation $\left(\theta_{\text {pref }}\right)$ for 9 connected LGN-V1 pairs, along with 63 simultaneously recorded, not-connected LGN cells. Most LGN cells had low OSI values, indicating a lack of significant orientation bias. However, we report the calculated values to illustrate that, under conditions during which V1 cells exhibit orientation tuning, simultaneously recorded LGN cells are only slightly biased for orientation. Even in rare cases when the LGN cell exhibits selectivity for orientation, its $\theta_{\text {pref }}$ is not similar to that of the target V1 cell. In the example of Figure $7 A$, the connected LGN cell exhibits some orientation bias $(\mathrm{OSI}=0.41)$, but its preferred angle $\left(-54^{\circ}\right)$ is 
A

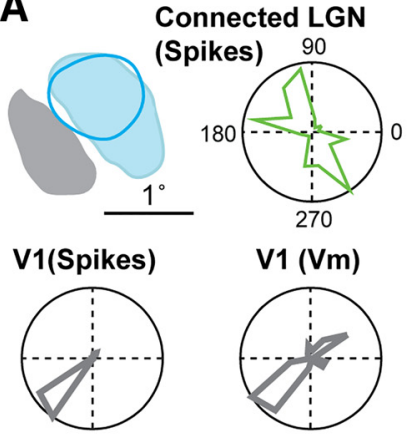

B

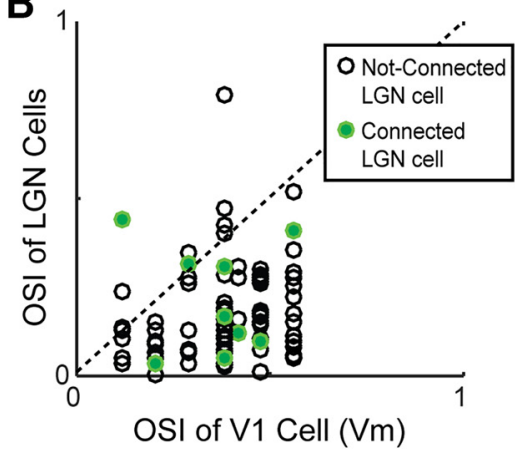

C

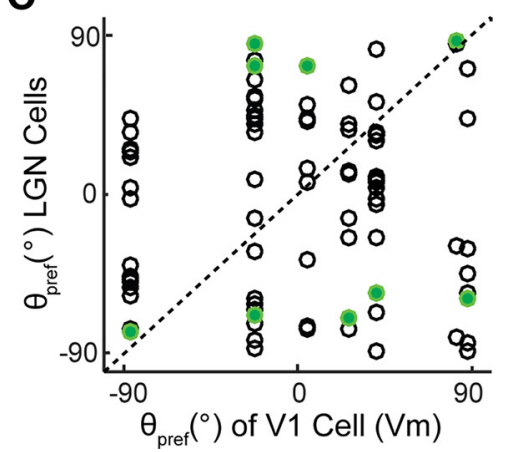

Figure 7. Orientation tuning similarity of connected LGN and V1 cells. $A$, Orientation tuning polar plots shown for a V1 cell and its presynaptic LGN cell, with RF overlap as indicated. Polar plots calculated from the V1 cell's spiking is similar to that calculated from its $V_{\mathrm{m}} \cdot \boldsymbol{B}$, Population plot of the OSI for connected (green) and not-connected (white) LGN cells plotted against the 0SI of the simultaneously recorded V1 cell. Larger OSI indicates higher selectivity. Dashes indicate line of equality. $\boldsymbol{C}$, Same as $\boldsymbol{B}$, but for the estimated preferred orientation ( $\left.\theta_{\text {pref }}\right)$.

nearly orthogonal to the preferred angle of the postsynaptic V1 cell $\left(\theta_{\text {pref }}=40^{\circ}, V_{\mathrm{m}}\right.$ OSI $=0.54$, spikes OSI $\left.=0.89\right)$.

In Figure $7 B$, we plot the OSI of LGN cells as a function of the OSI of the simultaneously recorded V1 cell. Connected LGN cells are shown separately (green). The range of OSI values for V1 is in line with previous reports of weaker orientation bias in $\mathrm{V} 1 V_{\mathrm{m}}$ responses compared with V1 spike responses (Carandini and Ferster, 2000). As expected, LGN cells had smaller OSIs than their simultaneously recorded V1 cell. More importantly, connected LGN cells did not have higher OSI values than nonconnected LGN cells (two-sample KS test).

In Figure $7 C$, we plot the $\theta_{\text {pref }}$ of LGN cells as a function of the $\theta_{\text {pref }}$ of the simultaneously recorded V1 cell. Similar to OSI, $\theta_{\text {pref }}$ of connected LGN cells was not close to the line of equality, and therefore not correlated with the $\theta_{\text {pref }}$ of the postsynaptic V1 cell (Fig. 7C). We conclude that, although cat LGN cells may exhibit orientation bias when driven at their optimal spatial and temporal frequency (Suematsu et al., 2012), they do not exhibit orientation bias aligned with that of the target V1 cell and thus cannot individually contribute orientation information to V1.

\section{Discussion}

We measured monosynaptic EPSPs generated by LGN cells in V1 cells of the anesthetized cat. The primary contribution of our work is the detailed characterization of geniculocortical EPSPs, which has immediate utility in deciphering an important circuit in systems neuroscience.

\section{EPSP features}

The mean EPSP amplitude was $0.42 \pm 0.26, \sim 5$ times smaller than in vitro estimates (Stratford et al., 1996), and closer to in vivo estimates from rat barrel cortex (Bruno and Sakmann, 2006). The discrepancy between in vitro and in vivo findings may be related to differences in the driving force, short-term synaptic depression (Boudreau and Ferster, 2005), and extracellular calcium concentration (Ohana and Sakmann, 1998; Massimini and Amzica, 2001), in the slice versus whole brain. The small amplitude and short duration $(9.7 \pm 4.2 \mathrm{~ms}$ ) of EPSPs reported here limit the window of integration for subsequent spikes. A previous study found a $7 \mathrm{~ms}$ window of facilitation between LGN spikes from two different neurons (Usrey et al., 2000), consistent with our observation of EPSP decay time constant of $7.9 \pm 8.4 \mathrm{~ms}$. The same study found a facilitation window of $15 \mathrm{~ms}$ between spikes from the same LGN cell. This is longer than the duration of EPSPs in our dataset, suggesting that this form of short-term facilitation relies on presynaptic mechanisms (Cardin et al., 2010).

Several factors may have influenced our EPSP amplitudes. First, the amplitudes we measure under anesthesia may be different from those measured during wake due to differences in LGN-V1 firing rates (Durand et al., 2016). However, comparison of our results to previous findings is justified because the only other intracellular study of thalamocortical EPSPs (Bruno and Sakmann, 2006), and previous extracellular studies, were also recorded in anesthetized states. A second factor is potential contamination of EPSPs by correlated spikes from other LGN cells. Given the fast rise time of the EPSP, only spikes that occur with $\sim 1$ ms synchrony would contaminate an existing EPSP. Previous work has shown that such tight synchrony occurs $0-40 \%$ of cases when two RFs are nearly identical, and is stimulus dependent (Alonso et al., 2008) but weak, with an average of 10\% (Yeh et al., 2009) or 28\% (Alonso et al., 1996) of synchronous spikes. To reduce contamination by coincident spikes, we measured EPSPs during response to white noise, a stimulus with low spatiotemporal correlations that produces low synchrony among neurons. Therefore, we believe that at most a small fraction $(10-28 \%)$ of coincident spikes may have contaminated our EPSP estimates. Accounting for an average overestimation of 19\% produces even smaller average EPSPs of $0.34 \pm 0.21$, across our dataset.

\section{Connections onto V1 RS and FS cells}

In vitro studies of rodent somatosensory cortex, and other sensory systems, have shown that thalamic axons in L4 make more numerous, and 2-4 times stronger, connections with FS interneurons than with RS pyramidal neurons (Porter et al., 2001; Cruikshank et al., 2007, Schiff and Reyes, 2012; Kloc and Maffei, 2014). In contrast, our data show similar EPSP amplitudes and average number of connected inputs for RS and FS cells. Furthermore, we found no signs of disynaptic inhibition in our STAs, suggesting that feedforward (disynaptic) inhibition is not stronger than feedforward (monosynaptic) excitation in input layers of cat V1.

Such differences in the strength of feedforward inhibition, between our in vivo characterization and prior in vitro characterization, may be due to inherent differences between slice and whole-animal studies, which may differentially affect RS and FS cells. For instance, in the mouse somatosensory cortex, a calcium-permeable AMPA receptor has been causally linked 
to larger EPSPs in L4 FS neurons (Hull et al., 2009). Differences in calcium concentration between in vitro and in vivo preparations could therefore differentially affect EPSPs in RS and FS cells.

In addition, the visual system of the cat has different functional demands compared with the somatosensory cortex of the rodent. Sensory responses in somatosensory cortex have short latencies, are brief, and generate few spikes, being limited strongly by powerful feedforward inhibition (Porter et al., 2001; Wehr and Zador, 2003; Gabernet et al., 2005; Sun et al., 2006; Higley and Contreras, 2007). In contrast, visual responses have longer latencies and durations, and even when responding to a brief stimulus generate larger spike outputs. This suggests a relatively weaker feedforward inhibition in the visual system of cats, consistent with our findings.

A notable difference between RS and FS V1 neurons in our study was the frequency of nonspecific connections, which occurred almost exclusively on FS cells (Fig. 6D). However, this difference in the specificity of LGN inputs does not impact either the orientation tuning (Cardin et al., 2007) or the linearity (Mata and Ringach, 2005) of the target V1 RF. Further studies are needed to determine the effect of nonspecific LGN input on feedforward excitation and inhibition in V1.

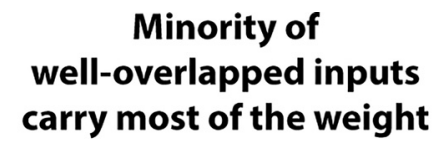

\section{Majority of inputs are well-overlapped and carry equal weight}
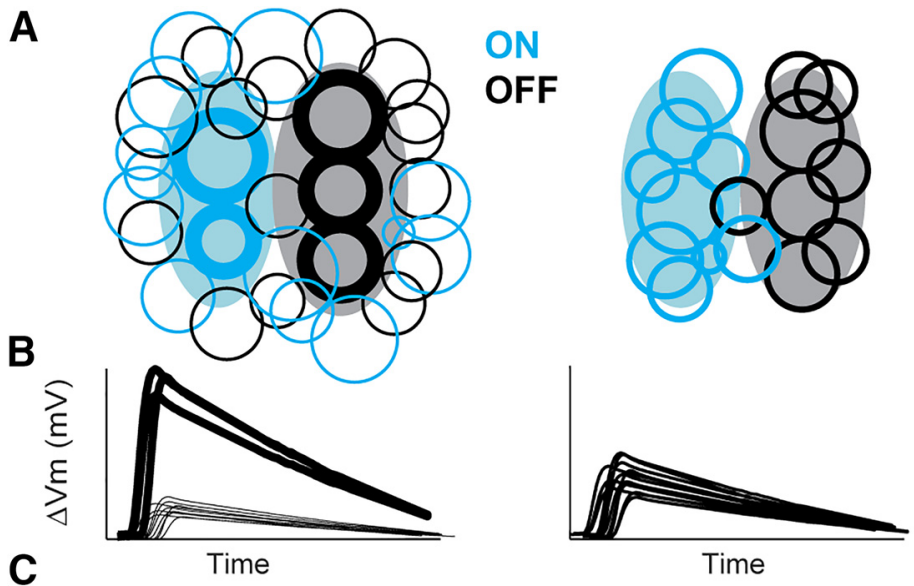

Time
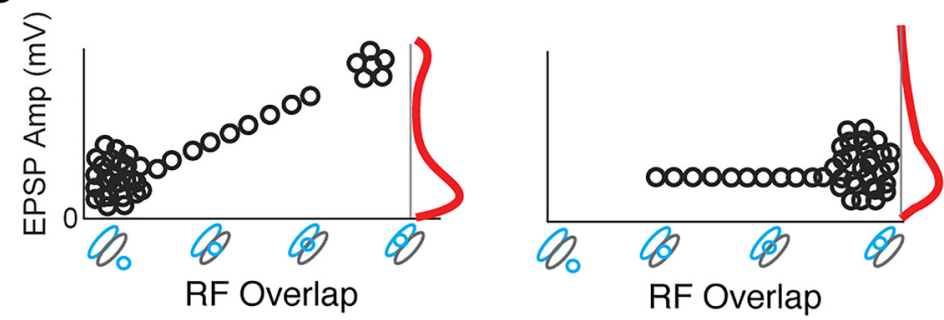

Figure 8. Schematized models of thalamocortical connectivity. Left, "Oligarchy" connectivity model where most connections have poor RF overlap and are weak. Right, "Democratic" connectivity model consistent with our observations, where most connections have good RF overlap and are equally weighted. L4 V1 simple cell spatial RFs are shown in filled elongated contours, and circular contours represent connected LGN RFs. $A$, LGN-V1 spatial RF contours for all LGN inputs to a V1 cell, with the synaptic strength represented in LGN contour thickness. $\boldsymbol{B}$, Corresponding EPSPS. C, Corresponding EPSP amplitude plotted as a function of LGN-V1 RF overlap, with the distribution of EPSP amplitudes shown in red.

\section{LGN-V1 response similarity and EPSP amplitude}

Three extracellular studies (Tanaka, 1983; Reid and Alonso, 1995; Alonso et al., 2001) provided support for Hubel and Wiesel's feedforward hypothesis (Hubel and Wiesel, 1962), showing precise RF overlap of connected LGN-V1 cell pairs. However, extracellular recordings only reported on spiking activity, and left open the possibility of abundant subthreshold connections with much less spatial specificity, similar to observations in the mouse visual cortex (Cossell et al., 2015). Indeed, the "feedforward" hypothesis, with precise wiring of LGN afferents to their cortical targets, is difficult to reconcile with the anatomy of the geniculocortical projection, with LGN axons terminating with large arborizations in L4 and hundreds of axons converging onto any point in V1 (Peters and Payne, 1993).

Intracellular recordings offer a more sensitive detection of synaptic connectivity and may reveal connections undetected in extracellular studies. Therefore, we expected to find a large subset of LGN inputs with small EPSP amplitudes and poor LGN-V1 RF overlap (Fig. 8A, left). Surprisingly, we did not find such connections, and our intracellular data largely agreed with the extracellular studies. We found that the probability of connection increases with increasing response and RF similarity and connections only occurred when there was spatial overlap between LGN-V1 RFs (Fig. 8A, right). This demonstrates a remarkable anatomical precision in the geniculocortical pathway, providing a validation of previous extracellular studies (Reid and Alonso, 1995; Alonso et al., 2001) and refuting the possibility that the extracellular connections were the tip of an iceberg of less specific, and weaker, synapses.

We expected to see a correlation between RF overlap and EPSP amplitude. In other words, consistent with Hebbian learning rules, we expected presynaptic (LGN) cells that are more likely to respond to the same visual stimulus as the postsynaptic (V1) cell to have a larger impact on the postsynaptic $V_{\mathrm{m}}$. Recent observations from intracortical connections in the mouse visual cortex were consistent with such expectations and reported a minority of cell pairs with the greatest RF similarity exhibiting the largest EPSPs (Cossell et al., 2015). Surprisingly, we did not find a correlation between either LGN-V1 RF overlap or response similarity and EPSP amplitude (Fig. 5).

The lack of such correlation shown in our data suggests a different mode of operation at the LGN to V1 synapse (Fig. 8). Rather than an "oligarchy" connectivity model in which few presynaptic inputs with high RF overlap contribute most of the synaptic strength (Fig. 8, left) as seen in intracortical circuits (Cossell et al., 2015), thalamocortical input is instead a "democratic" system in which all presynaptic LGN inputs with RF overlap contribute equally (Fig. 8, right).

Such a democratic connectivity scheme is consistent with the lack of small, weakly overlapped, LGN inputs in our dataset compared with those in prior extracellular studies (Alonso et al., 2001). Furthermore, as schematized in Figure $8 B$, it explains the relatively small range of the EPSP amplitudes in our dataset (range: $0.1-1.2 \mathrm{mV}$ ), compared with those reported from intracortical connections in mouse V1 (Cossell et al., 2015) (range: 
$\sim 0.1-3.5 \mathrm{mV}$ ). In the oligarchy model, a positive correlation can be seen between EPSP amplitude and RF overlap (Fig. 8C, left), due to the contribution of a minority of very large EPSPs from inputs with high RF overlap. In contrast, because the majority of RFs are well overlapped in the democratic connectivity model, and the range of their EPSP amplitudes are small, it follows that RF overlap and EPSP amplitude will not exhibit a correlation (Fig. $8 C$, right).

Previous work has shown that V1 firing is sensitive to the temporal dynamics of input LGN spikes (Usrey et al., 2000). Therefore, it is possible that firing of an L4 V1 neuron is determined by the joint input of many neurons, and small differences in thalamocortical EPSP amplitude of single inputs are irrelevant to the establishment of synaptic strength, leading to a democratic connectivity pattern. Another possibility is that homeostatic mechanisms serve to reduce the strength of frequent synaptic connections with high RF similarity relative to the more infrequent connections with slightly lower RF similarity. Novel technologies that allow the recording of many more presynaptic connections will help to determine the set of features, at the single input and population input level, that determine synaptic strength in the thalamocortical pathway.

\section{References}

Alonso JM, Swadlow HA (2005) Thalamocortical specificity and the synthesis of sensory cortical receptive fields. J Neurophysiol 94:26-32. CrossRef Medline

Alonso JM, Usrey WM, Reid RC (1996) Precisely correlated firing in cells of the lateral geniculate nucleus. Nature 383:815-819. CrossRef Medline

Alonso JM, Usrey WM, Reid RC (2001) Rules of connectivity between geniculate cells and simple cells in cat primary visual cortex. J Neurosci 21:4002-4015. Medline

Alonso JM, Yeh CI, Stoelzel CR (2008) Visual stimuli modulate precise synchronous firing within the thalamus. Thalamus Relat Syst 4:21-34. CrossRef Medline

Boudreau CE, Ferster D (2005) Short-term depression in thalamocortical synapses in cat primary visual cortex. J Neurosci 25:7179-7190. CrossRef Medline

Bruno RM, Sakmann B (2006) Cortex is driven by weak but synchronously active thalamocortical synapses. Science 312:1622-1627. CrossRef Medline

Carandini M, Ferster D (2000) Membrane potential and firing rate in cat primary visual cortex. J Neurosci 20:470-484. Medline

Cardin JA, Palmer LA, Contreras D (2007) Stimulus feature selectivity in excitatory and inhibitory neurons in primary visual cortex. J Neurosci 27:10333-10344. CrossRef Medline

Cardin JA, Kumbhani RD, Contreras D, Palmer LA (2010) Cellular mechanisms of temporal sensitivity in visualcortex neurons. J Neurosci 30: 3652-3662. CrossRef Medline

Contreras D, Palmer L (2003) Response to contrast of electrophysiologically defined cell classes in primary visual cortex. J Neurosci 23:6936-6945. Medline

Cossell L, Iacaruso MF, Muir DR, Houlton R, Sader EN, Ko H, Hofer SB, Mrsic-Flogel TD (2015) Functional organization of excitatory synaptic strength in primary visual cortex. Nature 518:399-403. CrossRef Medline

Cruikshank SJ, Lewis TJ, Connors BW (2007) Synaptic basis for intense thalamocortical activation of feedforward inhibitory cells in neocortex. Nat Neurosci 10:462-468. CrossRef Medline

da Costa NM, Martin KA (2011) How thalamus connects to spiny stellate cells in the cat's visual cortex. J Neurosci 31:2925-2937. CrossRef Medline

Durand S, Iyer R, Mizuseki K, de Vries S, Mihalas S, Reid RC (2016) A comparison of visual response properties in the lateral geniculate nucleus and primary visual cortex of awake and anesthetized mice. J Neurosci 36:12144-12156. CrossRef Medline

Freund TF, Martin KA, Whitteridge D (1985) Innervation of cat visual areas 17 and 18 by physiologically identified $x$ - and $y$ - type thalamic afferents. J Comp Neurol 242:263-274. CrossRef Medline

Gabernet L, Jadhav SP, Feldman DE, Carandini M, Scanziani M (2005) Somatosensory integration controlled by dynamic thalamocortical feedforward inhibition. Neuron 48:315-327. CrossRef Medline
Higley MJ, Contreras D (2007) Cellular mechanisms of suppressive interactions between somatosensory responses in vivo. J Neurophysiol 97:647658. CrossRef Medline

Hubel DH, Wiesel TN (1962) Receptive fields, binocular interaction and functional architecture in the cat's visual cortex. J Physiol 160:106-154. CrossRef Medline

Hull C, Isaacson JS, Scanziani M (2009) Postsynaptic mechanisms govern the differential excitation of cortical neurons by thalamic inputs. J Neurosci 29:9127-9136. CrossRef Medline

Humphrey AL, Sur M, Uhlrich DJ, Sherman SM (1985) Projection patterns of individual $x$ - and $y$-cell axons from the lateral geniculate nucleus to cortical area 17 in the cat. J Comp Neurol 233:159-189. CrossRef Medline Jones EG (1985) The thalamus. New York: Plenum.

Jones JP, Palmer LA (1987) An evaluation of the two-dimensional Gabor filter model of simple receptive fields in cat striate cortex. J Neurophysiol 58:1233-1258. Medline

Kloc M, Maffei A (2014) Target-specific properties of thalamocortical synapses onto layer 4 of mouse primary visual cortex. J Neurosci 34:1545515465. CrossRef Medline

Kondo S, Ohki K (2016) Laminar differences in the orientation selectivity of geniculate afferents in mouse primary visual cortex. Nat Neurosci 19: 316-319. CrossRef Medline

Massimini M, Amzica F (2001) Extracellular calcium fluctuations and intracellular potentials in the cortex during the slow sleep oscillation. J Neurophysiol 85:1346-1350. Medline

Mata ML, Ringach DL (2005) Spatial overlap of ON and OFF subregions and its relation to response modulation ratio in macaque primary visual cortex. J Neurophysiol 93:919-928. Medline

McCormick DA, Connors BW, Lighthall JW, Prince DA (1985) Comparative electrophysiology of pyramidal and sparsely spiny stellate neurons of the neocortex. J Neurophysiol 54:782-806. Medline

Nowak LG, Azouz R, Sanchez-Vives MV, Gray CM, McCormick DA (2003) Electrophysiological classes of cat primary visual cortical neurons in vivo as revealed by quantitative analyses. J Neurophysiol 89:15411566. Medline

Ohana O, Sakmann B (1998) Transmitter release modulation in nerve terminals of rat neocortical pyramidal cells by intracellular calcium buffers. J Physiol 513:135-148. CrossRef Medline

Peters A, Payne BR (1993) Numerical relationships between geniculocortical afferents and pyramidal cell modules in cat primary visual cortex. Cereb Cortex 3:69-78. CrossRef Medline

Porter JT, Johnson CK, Agmon A (2001) Diverse types of interneurons generate thalamus-evoked feedforward inhibition in the mouse barrel cortex. J Neurosci 21:2699-2710. Medline

Priebe NJ, Mechler F, Carandini M, Ferster D (2004) The contribution of spike threshold to the dichotomy of cortical simple and complex cells. Nat Neurosci 7:1113-1122. CrossRef Medline

Reid RC, Alonso JM (1995) Specificity of monosynaptic connections from thalamus to visual cortex. Nature 378:281-284. CrossRef Medline

Ringach DL, Hawken MJ, Shapley R (1997) Dynamics of orientation tuning in macaque primary visual cortex. Nature 387:281-284. CrossRef Medline

Sanderson KJ (1971) The projection of the visual field to the lateral geniculate and medial interlaminar nuclei in the cat. J Comp Neurol 143:101108. CrossRef Medline

Schiff ML, Reyes AD (2012) Characterization of thalamocortical responses of regular-spiking and fast-spiking neurons of the mouse auditory cortex in vitro and in silico. J Neurophysiol 107:1476-1488. CrossRef Medline

Scholl B, Tan AY, Corey J, Priebe NJ (2013) Emergence of orientation selectivity in the mammalian visual pathway. J Neurosci 33:10616-10624. CrossRef Medline

Shou TD, Leventhal AG (1989) Numerical relationships between geniculocortical afferents and pyramidal cell modules in cat primary visual cortex. J Neurosci 9:4287-4302. Medline

Skottun BC, De Valois RL, Grosof DH, Movshon JA, Albrecht DG, Bonds AB (1991) Classifying simple and complex cells on the basis of response modulation. Vision Res 31:1079-1086. CrossRef Medline

Steriade M, McCormick DA, Sejnowski TJ (1993) Thalamocortical oscillations in the sleeping and aroused brain. Science 262:679-685. CrossRef Medline

Stratford KJ, Tarczy-Hornoch K, Martin KA, Bannister NJ, Jack JJ (1996) 
Excitatory synaptic inputs to spiny stellate cells in cat visual cortex. Nature 382:258-261. CrossRef Medline

Suematsu N, Naito T, Sato H (2012) Relationship between orientation sensitivity and spatiotemporal receptive field structures of neurons in the cat lateral geniculate nucleus. Neural Netw 35:10-20. CrossRef Medline

Sun QQ, Huguenard JR, Prince DA (2006) Barrel cortex microcircuits: thalamocortical feedforward inhibition in spiny stellate cells is mediated by a small number of fast-spiking interneurons. J Neurosci 26:1219-1230. CrossRef Medline

Sun W, Tan Z, Mensh BD, Ji N (2016) Thalamus provides layer 4 of primary visual cortex with orientation- and direction-tuned inputs. Nat Neurosci 19:308-315. CrossRef Medline

Swadlow HA, Gusev AG (2002) Receptive-field construction in cortical inhibitory interneurons. Nat Neurosci 5:403-404. CrossRef Medline
Tanaka K (1983) Cross-correlation analysis of geniculostriate neuronal relationships in cats. J Neurophysiol 49:1303-1318. Medline

Usrey WM, Alonso JM, Reid RC (2000) Synaptic interactions between thalamic inputs to simple cells in cat visual cortex. J Neurosci 20:5461-5467. Medline

Vidyasagar TR, Jayakumar J, Lloyd E, Levichkina EV (2015) Subcortical orientation biases explain orientation selectivity of visual cortical cells. Physiol Rep 3:e12374. CrossRef Medline

Wehr M, Zador AM (2003) Balanced inhibition underlies tuning and sharpens spike timing in auditory cortex. Nature 426:442-446. CrossRef Medline

Yeh CI, Stoelzel CR, Weng C, Alonso JM (2009) Functional consequences of neuronal divergences within the retinogeniculate pathway. J Neurophysiol 101:2166-2185. CrossRef Medline 